\title{
Análisis de las relaciones entre el gobierno municipal y el desarrollo de la comunidad")
}

\author{
por \\ CARLOS MOUCHET \\ Profesor de la Universidad de Buenos Aires, Consejero de la Organización \\ del Plan Regulador de Buenos Aires, Ex-Dırector General de Asuntos \\ Legales de la Ciudad de Buenos Aires, etc.
}

\section{El PROBLEMA}

1. El desarrollo de la comunidad sobre áreas regionales o mer tropolutanas, en virtud de necesidades sociales y exigencias técnicas. Conflicto con las tendencias hacia una mayor autonomia municipal

El tema encierra una cuestión de mucho interés ante la necesidad de conciliar la organización poítica y juridica en diferentes niveies y sobre diversas jurisdicciones - propia especialmente de la organización federal-, con las necesidades de un buen desarrollo de la estructura de la comunidad, que no siempre se

(") Este trabajo fue presentado al II Seminario Universitario Interamericano sobre Asuntos Municipa'es, rea.izado en San Diego, Cal.fornia, EE. UU., entre e' 21 y e! 23 de octubre de 1960, inmediatamente después del VIII Congreso Interamericano de Municipios. Estuvo dedicado al estucio del a Desarrollo de la comunidado y se trataron dos temas básicos: 1) Diversos aspectos en la planeación del desarrollo de la comunidad; 2) El concepto federal en el desa:rollo de la comunidad. Este Seminario fue presidido por el Dr. Vincent L. Padgett, profesor de la Facultad de Ciencia Politica del eSan Diego State Colleges. 
orienta dentro de convencionales límites políticos y administrativos.

Esta cuestión afecta la llamada "autonomía» del Municipio. Un régimen federal sano exige una vida municipal rica, que sirva de sustento a las autonomías provinciales. Ciertas formas de desarrollo de la comunidad y las exigencias técnicas de la solución de sus problemas, crean situaciones criticas, tanto en los conceptos tradicionales del federalismo como de la autonomía municipal. El equilibrio económico y político que supone el federalismo, se rompe cuando uno de los grupos que lo integran crece en fuerza y densidad en forma tal que absorbe o domina a los demás. A su vez, el concepto de la autonomía municipal requiere nuevos enfoques cuando varios Municipios que representaban comunidades distintas se integran en un solo aglomerado social, que requiere el tratamiento conjunto de la solución de sus problemas, rompiendo en cierta forma el concepto de la autonomía local. Tal es, por ejemplo, el caso del área metropolitana de Buenos Aires, que es un factor de crisis de nuestro federalismo, y que obliga a colocar el examen de la cuestión municipal sobre otras bases diferentes de las tradicionales.

Gran parte de los problemas de desarrollo de la comunidad y de los servicios públicos que en otra época aparecían limitados al territorio de cada Municipio, ahora se plantean en forma cada. vez más creciente en términos de "áreas metropolitanas" y de "áreas regionales", con problemas comunes, y a su vez se está abriendo paso la política que considera los planeamientos regionales y metropolitanos dentro de un planeamiento general, a escala nacional (1).

(1) Ejemplo destacado de esta tendencia del planeamiento urbano regional dentro de un marco nacional es en el Brasil el conjunto de iniciativas y de proyectos llamado. «Operaçao Municipiò. Ver: Francelino de Araújo Gómez: (jpera̧̧o Municipio. Fundamento do plano nacional de obras e serviços municipais. Río de Janeiro, 1955; y Araújo Cavalcanti: Sentido e importancia de la Opera ción Rio de Janeiro, en aRevista Municipal Interamericanas, La Habana, enerojun:o de 1856. Sobre los problemas de las áreas metropolitanas en los EE. UU. de Norteamérica ver: Metropolitan Surveys: A digest, preparado por aGovernment 
Como nos vamos a referir especialmente al problema de Buenos Aires y su contorno el "Gran Buenos Aires", interesa definir previamente qué se entiende por "área metropolitana". Se entiende por tal el territorio que alberga un aglomerado humano perteneciente a varios Municipios, y que, social y económicamente, está integrado con una ciudad central. La vida entre la ciudad central y la población circundante se convierte en un todo de estrecha interdependencia, aunque no siempre "orgánico".

Como los problemas no se detienen en los límites de cada $\mathrm{Mu}$ nicipio, ello obliga a tratar los comunes a un área metropo!itana con un criterio de conjunto, lo que en principio supone regulaciones gubernativas y administrativas uniformes, o por lo menos coordinadas. Varias comunidades se integran de manera tal que forman una nueva y gran comunidad. Tampoco debe olvidarse, para medir la complejidad de los probiemas que aparecen, que aún dentro del área puramente comunal, la acción municipal sufre el impacto de las autoridades provinciales (estatales) y federales, en materia de servicios públicos, puertos, carreteras, aeropuertos, etc. Todas estas notas de un área metropolitana desde el punto de vista social, económico y técnico se dan en el caso de Buenos Aires y su contorno.

No solamente un área metropolitana -y la cuestión alcanza igualmente a las áreas regionales - puede desbordar los límites de un Municipio, sino que no siempre se encuentran en toda su extensión dentro de los limites de un Municipio, Estado o Provincia. Casos tipicos son en Estados Unidos los de Washington (que comprende el Distrito de Columbia y zonas pertenecientes a los Estados de Virginia y Maryland) y la de Nueva York (área que comprende territorios pertenecientes a Nueva York, Nueva Jersey y Connecticut). Y en la Argentina, la de Buenos A:res, que comprende el Distrito Federal y una parte del Territorio de da Provincia de Buenos Aires.

Las áreas metropo:itanas suelen dar origen a desajustes entre

Affairs Foundation», Ch:cago, 1959; y Jerome P. Pickard: Metropolitanization of the United States, Washington, 1959. 
las realidades humanas y sociales y la organización gubernativa y administrativa. Ha dicho Jordana de Pozas en un excelente estudio sobre el problema (2), que lo deseable sería que esas grandes concentraciones humanas se hallaran reconocidas por el Derecho como Entidades político-administrativas y disfrutaran de una organización adecuada a su extensión presente, a su desarrollo futuro probable, a las necesidades generales de su población, a sus fines propios y a su mejor ordenación y gobierno.

Para evitar las deficiencias y anormalidades que acompañan al desarrollo incontrolado de esos núcleos metropolitanos, la solución técnica aparentemente más deseable sería la unificación en una sola Entidad territorial, gubernativa y administrativa. Diversos factores poíticos y sociales se oponen abiertamente a esta solución. De ellos, quizás el de mayor peso es la resistencia de los Municipios a dejarse anexionar o absorber y, en los paises federales, la oposición de las Provincias o Estados ante todo proyecto que signifique privarles de territorio. Otro factor, que debe ponderar el hombre de gobierno lo constituye el exceso de concentración política y administrativa, que así se crearía, susceptible de producir desequiiibrios en la organización y vida política de un país. Pensamos en lo monstruosa que sería una ciudad como Buenos Aires - como ente politico y administrativo-, si se le agregaran los Municipios vecinos.

Ya no es posible sostener seriamente que la materia de la planificación -cuyo avance incontenible es fácil prever por su obvia necesidad-, pueda quedar limitada al perimetro de cada Municipio, sino que debe encuadrarse en una visión más general, en planes regionales y en el estudio a escala nacional. El desarrollo de los grandes centros urbanos depende, en muchos aspectos, de factores que no están a escala local únicamente.

Esta tendencia inspirada por consideraciones de orden social

(2) ILuis JoRdana de POzAs: Problemas de las grandes concentraciones urbanas desde el punto de vista de la organización administrativa, en eCrónica del I Congreso Iisero-Americano de Municipiosn, publicación del Instituto de Estudios de Administración Local, Madrid, 1959, págs. 83 y sigts. 
y técnico, parecería estar en colisión con las aspiraciones hacia una mayor autonomía municipal, que constituye lo fundamental del programa de la intermunicipalidad americana. Los $\mathrm{Mu}$ nicipios ven con inquietud, como un peligro, la posibilidad de la creación de superorganismos de planificación regionales que mermen la autonomía municipal. $Y$ las Provincias y Estados locales de los paises federales miran esas perspectivas también como factores de nuevas debilitaciones del federalismo.

En los Congresos Interamericanos de Municipios se han planteado dos problemas: 1) El del planeamiento urbano con una visión a escala regionai y aún nacional, y 2) El de la preservación de los poderes y atribuciones de 'os Municipios. En el Congreso Interamericano de Municipios reunido en Río de Janeiro en 1958, después de admitirse que el desarrollo de los actuales procesos tecnológicos y de la economía de las grandes empresas, tienden a transferir al plano regional o nacional servicios básicos de utilidad pública, como energía eléctrica, abastecimiento de agua, etc., declaró que "de acuerdo con la organización política y administrativa de cada país, esos organismos podrían tener o no carácter intermunicipal, debiendo ser siempre considerados en esos planes los intereses y el respeto de las autonomías locacales", y que para la solución de esos problemas "debe intens:ficarse el régimen de acuerdos y convenios intermunicipales entre los Municipios y las demás esferas de gobierno, así como la consulta popular en los casos que se juzgue convenienten.

En el Primer Seminario Interamericano de Estudios Municipales realizado también en 1958 en San Pablo (Brasil), se afrontó en forma muy clara la cuestión. Se declaró que "al Munic1pio debe participar en el planeamiento regional por medio de consorcios o de convenios con las autoridades de los gobiernos nacionales, de los Estados-miembros o Provincias, o entre los Municipios interesados"; que "cuando se trata de planeamiento regional debe entenderse lo regional en términos de intermunicipalidad"; que "la metodología de la planificación regional deberá atender a los problemas de competencia derivados de los di- 
versos tipos de Estado, fijándose las normas generales o programáticas por parte de los gobiernos nacionales" (3).

En lineas generales, esa resolución coincide con la aprobada en el Congreso Hispano-luso-americano-filipino de Municipios reunido en Lisboa en Mayo de 1958 (4). Se resolvió que era de cla competencia del Municipion la elaboración del planeamiento urbano y la realización de su correspondiente ordenamiento urbano dentro de su área adm:nistrativa, y que en el caso que su expansión urbana origine la conveniencia de un planeamiento regional, y éste se realice con intervención del Estado, "es indispensabie que en todas las etapas de su elaboración se verifique la presencia efectiva de los Municipios interesados, en especial del Municipio de la aglomeración más desarrollada, que ha de ser el centro de más importancia regionaln. También se declaró, como altamente recomendable, que "los planeamientos regionales fueran de preferencia elaborados por las federaciones o agrupaciones de Municipios, siempre que reúnan un mínimo de capacidad técnica y económica, con la orientación y la presencia efectiva del Estado para la solución de los problemas de escala nacionaln.

Como se ve, se espera la preservación de la autonomía municipal, conciliándola con las necesidades de la planificación, mediante el régimen de acuerdos. La solución es especialmente recomendable en los países de organización federal, pues también significa el respeto de la autonomía provincial por el poder central nacional, ya que esos acuerdos requieren la presencia de los gobiernos provinciales o estatales.

El desarrollo de las comunidades en las áreas metropolitanas y regionales ob'iga a mirar la cuestón de la llamada autonomía municipal desde nuevos enfoques. Son ilustrativas a este respecto las conclusiones del Primer Seminario sobre problemas de urbanización en América latina, que bajo el patrocinio de las $\mathrm{Na}$ ciones Unidas se realizó en Santiago de Chile en julio de 1959.

(3) Primer Seminario Interamericano de Estudios Municipales, en Revista Municipal Interamericanas, La Habana, ju'io d:c:embre de 1956. núms. 1-2, pág. 27.

(4) Revista Municipal», núm. 81, año 1959, Lisboa (publicación oficial del Municipio de Lisboa). 
Se llegó a la conclusión de que las aspiraciones de los Municipios hacia una mayor autonomía no debían tener un sentido limitado a lo político. Debe tener un sentido de descentralización técnica, dentro de planes regionales y aún nacionales de coordinación. $\mathrm{Y}$ esta dea es aplicable en muchos aspectos dentro del régimen federal a las Provincias (5). En esa reunión se propuso como conveniente la acción armónica entre los distintos niveles de gobierno mediante la existencia en el gobierno central de un organismo permanente de coordinación especificamente encargado de los problemas de urbanización, a fin de examinarlos en conjunto, sin menoscabo de la iniciativa y competencia de las autoridades locales y provinciales (6).

2. El desarrollo de las comunidades locales y metropolitanas en los paises de régimen unitario y de régimen federal

La legislación sobre planificación del desarrollo ordenado de la comunidad es diferente en cuanto a su alcance en los paises de organización unitaria y en los países federales. En los segundos ofrece dificultades conjugar la planificación en los tres grados -federal, provincial y municipal-. Estas dificultades tienen menor importancia en los países centralizados (7).

Recordemos en primer término el ejemplo de Inglaterra, que atrae tanto el interés de los estudiosos. La Tozm and Country Planning Act de 1947, de carácter nacional, según su propio preámbulo, tiene como propósito la planificación en todo el pais

(5) Ricardo Zorraquf́ Becú sostiene que el problema del feceralismo argentino debe resolverse fuera de los moldes exc'usivamente juridicos. aLas Provi"c'as -dice- ya no son organismos adecuados para lograr una obra de gobierno ef:caz, y sus fronteras son limites irreales de politica tanto económica como socialp. Las Provincias deben cumplir nuevas funciones en un régimen de descentralización de !a actividad gubernativa (El federalismo argentino, Buenos Aires, 3.a ed. 19j8, páginas 230 y sigts.).

(6) Nacionfs Unidas: Informe provisional del Seminario sobre problemas de „rbanización en América latina, 195̄9, pág. 87.

(7) Cfr. Anton:o Delorenzo Neto: A planifição municipal, en aRevista dc Serviço Publicos, Río de Janeiro, abril de $195 \%$. 
del desarrollo urbanístico y del uso de la tierra. Prevé la existencia de planes de desarrollo a formularse por autoridades locales de planeamiento que ejercen jurisdicción sobre zonas con problemas comunes, vinculando en tales casos a varios Condados en un Distrito común (art. $1 .^{\circ}$, inc. $2 .^{\circ}$ y art. $5 .^{\circ}$ ). Se forman planes territoria'es, cuyo perímetro forma el Ministerio del ramo en su función urbanística (art. 5..$^{\circ}$. Hay también planes comunales (artículos $70^{\circ}$ y 13 ) y de conjuntos comunales que pueden abarcar grandes radios metropolitanos.

En el "Greater London", junto al Condado de Londres y a la City, existen tres Burgo-condados, 36 Burgos municipales, 26 Distritos urbanos, tres Distritos rurales, tres Parroquias, y cinco Condados, sin contar otras autoridades. La referida Ley (artículo 114), crea una autoridad de planeamiento para el área metropolitana de Londres.

Para valorar debidamente las peculiaridades de la legislación inglesa sobre planificación, debemos tener presente no sólo su carácter nacional, sino también la circunstancia de que Inglaterra no posee una Constitución escrita. El Parlamento inglés, al dictar legislación sobre la materia actúa a la vez como órgano constituyente y como órgano legislativo, de tal manera que por una ley puede introducir moditicaciones en la estructura gubernativa del pais y afectar o modificar el derecho de propiedad.

En los paises federales, las regulaciones sobre esta materia quedan sometidas a las legislaciones provinciales o estatales, aunque se está abriendo paso la idea de que también cabe al gobierno federal una función rectora, por lo menos para la coordinación y el asesoramiento, en los aspectos que desbordan lo local.

En los Estados Unidos, la legislación sobre planeamiento u ordenamiento urbano se considera materia de competencia propia de las legisiaturas locales, salvo aquellos aspectos que se refieran a materias interjurisdiccionales (8).

(8) Ver Comparative Digest of Municipal and Country Zoning Enabling Statutes, Washington, 1952. 
Tomando como ejemplo típico la General Municipal Law del Estado de Nueva York, señalemos que la misma prevé la existencia de planes para el desarrollo de áreas regionales, capaces de incluir Condados, Municipios y áreas metropo'itanas (art. 223-B y D). Establece además la implantación obligatoria de planes directores para los grandes centros urbanos (art. 28-A).

Para el desarrollo del área metropolitana de Nueva York que afecta tres Estados, existen dos organismos: el "Metropolitan Regional Council" y el "Metropolitan Council at Planning Agencies". Según deducimos de una reciente información aparecida en el Boletín informativo del "Department of City Planning" de la ciudad de Nueva York, el Consejo regional se encuentra en pleno proceso de organización, habiendo aprobado en su reunión del 23 de febrero de 1960, la elaboración de un estatuto legal y la provisión de medios financieros de que carecía hasta ahora

I a Constitución federal del Brasil nada establece sobre la competencia para legislar sobre el desarrollo en materia urbanística. Hay autores que consideran que el gobierno federal podría y debería dictar un Código Nacional de Urbanismo, en armonía con el Código Nacional de la Salud y el Plan Nacional de Vialidad, de acuerdo con previsiones de la Constitución federal (artículo 5. ${ }^{\circ}$, núm. XV) (9).

Brasil es el único país de América cuya Constitución nacional garantiza verdaderamente a los Municipios una serie de facultades propias no sujetas a las contingencias y variaciones de la legislación (art. 28). Se ha llevado a sus últimos extremos el sistema federalista, pues los Municipios están frente a los Estados en la misma relación que éstos con la Unión federal (10).

En los países de sistema federal, el respeto de las autonomías provinciales y municipales exige que la solución de los problemas de áreas regionales y metropo'itanas se verifique mediante el sistema de los acuerdos entre las autoridades federales,

(9) Hely López Meirelles: Limilaçóes urbanisticas ao uso de la propiedade, en Revista dos Tribunaiso, vol. 281, marzo de 1959, págs. 15 y sigts.

(10) Ver Carlos Mouchet: Facultades legislativas y reglampntarias de los Musnicipios, en revista alla Leyw, Buenos Aires, 1959, tomo 95, págs. 889 y sigts. 
provinciales y municipales. Veremos que ésta es la solución para Buenos Aires.

Ello no impide que el gobierno federal, dentro de ciertos límites, pueda cumplir una labor de asesoramiento y coordinación, sin perjudicar las autonomías locales.

En los Estados Unidos suscita serias dudas, por razones constitucionales, la posibilidad de la creación de un órgano nacional de planificación. Sin embargo, ante el desarrollo creciente de las áreas metropolitanas y la necesidad de estudiar sus problemas en escala apropiada, algunas personas responsables de ese pais me han expresado su opinión favorable a la creación de un organismo nacional para la materia, aunque con facultades limitadas al asesoramiento para mejor coordinar las actividades federales, estatales y municipales en todo lo que se refiera al des: arrollo de los centros urbanos y rurales, y sin vulnerar los poderes de los Estados.

Opino que nada se opondría también a una solución de ese tipo en la República Argentina.

II. Papel del Municipio en la Argentina conforme al sistema FEDERAL DE GOBIERNO

3. El régimen municipal en la Constitución nacional. Relaciones entre Municipio, Provincia y Estado nacional

En la Argentina el artículo 5. ${ }^{\circ}$ de la Constitución nacional impone a las Provincias dictar una Constitución que "asegure. su régimen ínuicipaln.

Esta fórmula, si bien significa el reconocimiento de la existencia natural y preexistente de las Entidades municipales, y la necesidad de que posean un régimen propio, deja, en cambio, en libertad a las Provincias de determinar la estructura de la organización municipal. Por ello ha podido exponer. la Corte Suprema de Justicia un concepto restringido, puramente legalista del Municipio, al declarar que "las municipalidades no son más que delegaciones de los poderes provinciales, circunscriptas a fines y li- 
mites administrativos, que la Constitución ha previsto como Entidades del régimen municipal” ("Fallos", t. 114, pág. 282 y tomo 123, pág. 313). De este concepto estrictamente jurídico está ausente la consideración de la realidad histórica y natural del Municipio. El Municipio parece nacer así, ex novo, por la creación de una ley provincial, lo que no se ajusta a la idea de que el Municipio es una institución natural y necesaria. Es la misma teoria de la Corte norteamericana, expuesta en numerosos fallos.

Según este criterio, que confirma el Derecho público argentino sobre la materia, tal como lo interpreta el más Alto Tribunal del pais, las municipalidades no tienen poderes legislativos. propios, sino meramente delegados para asuntos de Administración local y en un orden de reglamentación de las atribuciones que le confieren las leyes orgánicas.

Los autores argentinos han asignado una gran función al $\mathrm{Mu}$ nicipio dentro de la organización federativa. Ambas instituciones, unidas a la división de los poderes, representan una organización que evita la concentración del poder en una sola parte del cuerpo político (11). Ambas son formas de descentralización que contribuyen a proteger la libertad de los ciudadanos y a establecer una buena división del trabajo en la actividad gubernativa y en el impulso del desarrollo del pueblo. Lo fundamental es quecrean, al lado de las variadas formas de asociación que se dan en la vida social y partiendo de la familia, sociedades intermedias entre el individuo y el Estado.

Asi, para el gran Estrada, las ideas de municipalismo y de federalismo son inseparables, dentro de su concepción politica, como medios de descentralización del poder tendentes a asegu-

(11) No todos los autores piensan que el federal:smo considerado en forma abstracta sea una garantia de la libertad politica. Ver: Franz L. Neunann: Federalismo y libertad: una crítica, en la colección de estudios titulado aPráctica del federalismos, reunidos por Arthur W. Mac Mahon, Buenos Aires 1959, capítulos 64 y siguientes. En contra de esta op:nión recordemos la idea de KEL SEN, quien afirma que la descent:alización permite una aproximación mayor a la idea de la democracia. Esta idea es e! principio de la autoceterminación». También seña!a que alas autocracias revelan una preferencia natural por la centralización estáticad (Hans KELSEN: Teoría general del Derecho y del Estado. \$räducción de E. García Maynez. México, 1958, págs. 370-371. 
rar la libertad. "La noción de Provincia -expresa- confirma y completa la noción del Municipio; la naturaleza y el papel de ambos son explicados por la misma ley y responden a un mismo ideal" (12). Para él, Municipio, Provincia y Nación son tres categorías políticas o tres Entidades autonómicas con gobierno propio.

En la Argentina se perfila una tendencia a dar mayor autonomía a los Municipios. Sin esperar una reforma que asegure en la Constitución nacional esa autonomía, en la esfera provincial se han hecho algunos ensayos para dar mayor plenitud al gobierno propio municipal, mediante el sistema de la Carta. Este sistema llegó a tener vida, aunque corta, en la Provincia de Santa $\mathrm{Fe}$, cuya Constitución de 1921, puesta en vigencia en 1932, y abolida en 1935, la establecía para los Municipios de más de $\mathbf{2 5 . 0 0 0}$ habitantes. Esta corriente en favor de las Cartas municipales ha reaparecido en la Argentina con motivo de la sanción de las Constituciones de las siete nuevas Provincias argentinas. El principio se ha incorporado expresamente a las Constituciones de Río Negro (arts. 176/177), Formosa (art. 141) y Neuquén (art. 182) (13).

Pero es necesario insistir en la idea de que mientras la Constitución nacional no asegure expresamente la autonomía municipal, garantizando sus bases po:íticas, administrativas y financieras, ella quedará sujeta a las variaciones del Derecho de cada Provincia (14).

En el sistema actual, las municipalidades tienen relaciones con el gobierno federal a través de los gobiernos provinciales, y necesitan la conformidad de éstos para celebrar acuerdos con aquél (por ejemplo, art. 43 de la Ley orgánica de las Municipalidades de la Provincia de Buenos Aires).

(12) José Manuel Estrada: Curso de Derecho constitucional, t. II, en obras completas», Buenos Aires, 1902, t. IV, pág. 347.

(13) Ver Salvador M. Dana Montaño: La primera Constitución de la Provincia de Rio Negro, en revista eLa Leys, t. 90, pág. 719.

(14) Ver A. P. Borrajo, Alberto Elguera, C. Mouchet, R. Tibiletri, V. S. VIN8นL1 y C. E. WeIss: El régimen municipal en la Constitución. Buenos Aires, 1959. 
Los autores de la Constitución federal de $\mathbf{1 8 5 3}$ no consideraron conveniente dar normas uniformes para la organización de los Municipios en todo el país (15). Se inspiraron en la pureza de los principios federalistas. Pero la experiencia recogida desde entonces indica la conveniencia de establecer como garantía del Municipio las bases mínimas de su organización y facultades, así como previsiones para la coordinación de su actividad con la de otros niveles de gobierno.

III. El régimen municipal de la ciudad de Buenos Aires, CAPITAL FEDERAL DE LA RePÚbLICA

4. La Constitución nacional y el gobierno municipal de la ciudad de Buenos Aires. Relaciones con el gobierno federal

La institución del régimen municipal ha sido establecida en la Constitución federal como un requisito para que a las Provincias se les garantice la autonomía, pero lamentablemente en ella no se ha definido tal régimen ni enumerado las facultades y recursos de los Municipios, con lo que se ha favorecido los procesos de centralización.

Las imprecisiones y las dudas aumentan cuando se trata de determinar el régimen municipal de la ciudad de Buenos Aires, hasta tal punto que hay una corriente de opinión doctrinaria que ha llegado, en su expresión extrema, a negar a los vecinos de la ciudad de Buenos Aires que tengan constitucionalmente el derecho a un régimen municipal, pudiendo ser gobernada en la misma forma que Washington.

Prescindiendo de las discusiones doctrinarias existentes, 1o cierto y real es, que hasta ahora el gobierno nacional se ha atribuído, en virtud de lo dispuesto en los artículos 67, inc. $27, \mathrm{y}$ 86 , inc. tres, de la Constitución nacional, la facultad de re-

(15) Ver Carlos Motchet: Las idcas de Echeverria, de Alberdi y de los constituyentes de $13 ; 3$ sobre el régimen matricipa!, en rev. oLa I.ey, $t$. S3, liuenos Aire:. año 19056 . 
gular el régimen de gobierno y administración local de la ciudad de Buenos Aires.

Desde luego, debe descartarse por las mismas razones aplicables a los. Municipios de las Provincias, la existencia de una verdadera autonomía para la Municipalidad de ị ciudad de Buenos Aires, frente al gobierno federal. No ejerce sino la autoridad que le delega el Congreso de la Nación, y que puede variar según la voluntad de este órgano. Este criterio -ajustado a la legalidad - no es inconciliable con la tesis de la existencia necesaria del Municipio de la capital federal, en virtud de los principios del Derecho natural y de la interpretación de la Constitución (16): Así, el iegislador si bien podría regular esta institución, estableciendo por ejemplo un tipo de gobierno municipal diferente del actual, o aumentar o disminuir sus facultades, no podría suprimir la institución ni tampoco dejar de dotar a la misma del mínimo de facultades necesario para cumplir'su misión de velar por los intereses locales.

\section{Organización y atribuciones}

La Ley orgánica vigente núm. 1.260 (que dạta de 1882 y ha sufrido algunas modificaciones), organiza el gobierno y la administración de la ciudad, dividido en una rama ejecutiva, cuyo tiular es el Intendente municipal, designado por el Presidente de la Nación, y una rama deliberativa de elección popular, denominada Concejo deliberante. Este régimen es totalmente centralizado, ya que los poderes de dichos órganos se extienden sobre toda la extensión de la ciudad, sin ninguna forma de descentralización ni siquiera de carácter administrativo.

La Municipalidad tiene facultades en materia de poderes de policia, impositivos, servicios públicos, disposición y administración de sus bienes, etc.

El destino de este Municipio ha sufrido muchas vicisitudes y

(16) Máximo I. Gómez Forgues: La Municipalidad dẹ Buenos Aires y la reforma constitucional de 1949, Buenos Aires, $19 \overline{2} 2$. 
complicaciones por ser Buenos Aires la ciudad más importante del país y sede de las autoridades centrales, creando una tensa convivencia cuando estas se han sentido afectadas en sus prerrogativas o en su prestigio. La rama popular de la Municipalidad ha sufrido por ello, varias veces, arbitraria disolución.

IV. EI. DESARRollo DE LA COMUNidAd EN EI Área METROPOLItana de Buenos Aires

6. Naturaleza del área metropolitana de Buenos Aires. Sus problemas políticos, sociales, administrativos y técnicos

Hemos dicho que todas las notas de un área metropolitara desde el punto de vista físico, social, económico, de la circulación y transportes, etc., se dan en el área formada por Buenos Aircs y la zona circundante, con los consiguientes problemás técnicos, institucionales y jurídicos para encauzar su desarrollo.

En el Primer Congreso Nacional de Asuntos Municipales reunido en Buenos Aires en junio de 1960, en el cual actuamos como Secretario técnico, se señaló que Buenos Aires cumple actualmente las siguientes funciones: 1) Puerta principal de entrada de la Nación. 2) Principal centro de convergencia industrial. 3) Canglomerado urbano de mayor número de habitantes. 4) Centro financiero. 5) Centro cultural. 6) Centro administrativo nacional. La concurrencia de estas funciones ha dado lugar a una enorme extensión urbana, y consiguientemente a la existencia de una zona suburbana en continuo crecimiento.

Ese desarrollo, favorecido por la capitalidad de la ciudad de Buenos Aires, por la convergencia de las redes ferroviarias, viales y energéticas, ha sido incontrolado e inorgánico. Aparte de los problemas que ha creado en su propio territorio, ese área constituye un factor de desequilibrio político, económico y social con réación al resto del país. En efecto, ?uede calcularse que este área alberga $\mathbf{6 . 5 0 0 . 0 0 0}$ habitantes. Esto significa una 
proporción muy grande con relación a la población total del país de 20.000.000. Esta hipertrofia de Buenos Aires constituye ano de los factores más decisivos en la crisis del federalismo argentino (17).

En el aspecto institucional y legal, el área metropolitana de Buenos Aires presenta la particularidad y la dificultad de constituir un área interjurisdiccional con dos zonas sometidas en principio a dos jurisdicciones políticas y administrativas de grado superior: 1) La zona de la capital federal, sede del Gobierno Nacional Federal, sujeta a una legislación que dicta el Congreso federal como legislatura nacional o local, según las materias, y que ha organizado en el segundo aspecto una Municipalidad, con un repertorio de atribuciones delegadas. 2) La zona del "Gran Buenos Aires", sujeta a la jurisdicción de la Provincia de Buenos Aires (con autonomía provincial), y que comprende un conjunto de Municipalidades con poderes otorgados por la Constitución provincial y la Ley orgánica de Municipalidades. Además, sobre todo este conjunto territorial, el Congreso federal dicta legislación federal interestatal (por ejemplo: en materia de servicios públicos).

Hasta ahora, estas diversas autoridades no actúan en conjunto, de acuerdo con planes y métodos orgánicos para afrontar los problemas comunes a toda la zona. Puede hablarse también de una zona o área de influencia regional de Buenos Aires, que se extiende a unos cien kilómetros de la ciudad de Buenos Aires y el área La Plata-Berisso-Ensenada, que a su vez constituye un área metropolitana menor.

7. Soluciones para el desarrollo ordenado del área metropolitana de Buenos Aires.

Existe ya la comprensión de que no puede realizarse el planeamiento de la ciudad de Buenos Aires, definiéndolo únicamente dentro de sus límites, porque esta ciudad forma parte de una unidad territorial y de un conglomerado humano más vas-

(17) Ver Ricardo Zorraquf́ Becú: op. cit. págs. 223 y sigts. 
to, que se asienta sobre un territorio de similares condiciones desarrollada en una estrecha interdependencia.

Todo su ámbito debe someterse a un criterio integral, con previsiones y soluciones de conjunto.

En 1944, el Decreto-ley 9.434/1944, por el que se facultó a la Municipalidad a establecer la zonificación de la ciudad y restricciones al dominio, previo un principio de regulación conjunta. del área metropolitana, al facultar a dicha Municipalidad para celebrar "con los Municipios de la Provincia de Buenos Aires los convenios que juzgue convenientes para completar y extender la división en zonas autorizadas por este Decreto o acordar la respectiva cooperación, a la que a su vez se establezcan en ellos". Como se ve, la previsión -que no se puso en práctica- se limita a uno sólo de los aspectos de la posible regulación conjunta, es decir, a la zonificación. No teniendo imperium sobre el territorio de la Provincia de Buenos Aires, el Decreto-ley se limitó a abrir la puerta a los acuerdos interjurisdiccionales.

Actualmente, se considera que la Municipalidad de la ciudad de Buenos Aires, representativa del núcleo más importante dentro del área metropolitana y contando con medios técnicos y financieros, puede tomar, como lo ha hecho, la iniciativa del planeamiento metropolitano y regional.

El camino lo ha señalado la Ordenanza municipal número 14.637 del año 1958, que creó el “Estudio del Plan Regulador de la Ciudad de Buenos Aires", y lo autorizó a proponer las bases de acuerdos con la Provincia de Buenos Aires y con los organismos del Gobierno federal.

Es política de acuerdos, ya iniciada, es la más adecuada a la organización federal del país, a nuestra tradición política y al sentido democrático del pueblo argentino.

Otras soluciones, como la creación de un superdistrito fedcral encontrarian objeciones de carácter constitucional y resistencias de tipo político, ya que tendría que hacerse a expensas de la Provincia de Buenos Aires, por la absorción y anexión a la capital federal de varios Municipios. Además, agravaria la ya excesiva concentración actual. Por lo tanto, debe desecharse una solución de esta naturaleza. 
También una solución institucional y jurídica de vastos alcances podría surgir de una reforma de la Constitución nacional que autorizara al Gobierno nacional la preparación y aplicación de un Plan regulador para el área metropolitana de Buenos Aires y el establecimiento de un tribunal técnico jurisdiccional para hacerlo cumplir (18). Esta solución tropezaria con la objeción fundada en que sacrificaría aspectos de la autonomía provincial y prescindiría de la intervención plena. de las Municipalidades.

La política de los acuerdos es la única que se adecúa a nuestra organización constitucional y que puede originar la confiada cooperación y concentración de esfuerzos de las diversas Entidades politicas afectadas. Por ello, la Municipalidad de Buenos Aires ha propuesto hace unos meses acuerdos al Gobierno nacional y a la Provincia de Buenos Aires, para coordinar la labor conjunta de planeamiento del desarrollo de las zonas urbanas, rurales y de reserva, tanto en el conglomerado metropolitano, como en el área regional Buenos Aires-La Plata.

Los problemas del área metropolitana de Buenos Aires merecieron una detenicla consideración en el Primer Congreso $\mathrm{Na-}$ cional de Asuntos Municipales, ya recordado, reunido en la capital federal en junio de 1960. Fueron encarados a escala nacional y regional.

Para contener la hipertrofia de la estructura física del área metropolitana, se recomendó estudiar la planificación orgánica de las redes circulatorias y energéticas del país, del sistema portuario, de la industria, etc., aplicando el criterio regional para el desarrollo integral de la misma.

Como se planteó la cuestión de la incidencia, que podría tener el carácter de capital de la ciudad de Buenos Aires, se llegó a la conclusión de que la conveniencia de mantener o no esa capitalidad surgiría de los estudios de planificación en escala nacional.

(18) Esta es una de las alternativas expuestas en el estudio titulado El régimen municipal de la Constitución, de A. P. Borrajo, A. Elguera, C. Mouchet, R. TIbiletti, V. S. Vinellr y C. E. Weiss, ya citado, pígs. 78 y sigts. 
En cuanto al planeamiento del desarrollo del área metropolitana de Buenos Aires, se hicieron las siguientes declaraciones: 1) "Que las comunas que integran el área metropolitana de Buenos Aires y su zona rural de influencia deben consolidar y prever la continuidad de los medios juridicos y técnicos organizativos para encarar en conjunto la resolución de los problemas del área total, respetando las autonomías jurisdiccionales de acuerdo con una estructura previamente aceptada en forma conjunta". 2) "Que los planes reguladores que deben formular. las comunas participantes en el acuerdo mencionado se desarrollen siguiendo los alineamientos generales emergentes de la solución que en forma común se acepte" (19).

La política de planeamiento del desarrollo del área metropolitana de Buenos Aires, de acuerdo con los antecedentes reccrdados existentes en países de la misma estructura política y gubernamental que el nuestro (como los Estados Unidos), y siguiendo el método de los acuerdos ya propiciado e iniciado, debe culminar en la creación de una Junta para el área metropolitana y regional de Buenos Aires, como organismo de asesoramiento y coordinación, formada de la manera que se determinaria oportunamente, con representantes de la Municipalidad de Buenos Aires, de los Municipios de la Provincia de Buenos Aires, comprendidos dentro de la zona inetropolitana (y aún de la zona de influencia regional), del Gobierno nacional y reparticiones autárquicas nacionales y del Gobierno de la Provincia de Buenos Aires. Deberia tener por funciones promover un plan regional, controlar su efectuación, aparte de otras importantes tareas como la de asesorar en materia de prioridades y en materia de obras y de servicios públicos, que afectaran el área o región de planeamiento conjunto. Dadas sus funciones puramente de asesoramiento y coordinación, no afectaría la autonomía de la Provincia de Buenos Aires ni la de los Municipios comprendidos en el área, y permitiría a cada una de estas Entidades ejecutar la parte correspondiente de una p'anificación conjunta.

(19) En este Congreso fue Relator del tema III CPaneamiento y Viviendas, e' arquitecto Eduardo J. Sarrailh, Consejero Ejecutivo del Plan Regulacor de la Ciudad de Buenos Aires. 
V. Papel del Gobierno federal en la coordinación con las Provincias Y Municipios del DESARROllo de las COMUnidades LOCALES Y DE LAS ÁREAS METROPOLITANAS Y REGIONALES

8. Los procesos sociales y económicos del mundo contemporanco obligan a trazar planes que abarcan áreas que en el interior de un pais no se adaptan a los limites, a veces convencionales, de las Provincias y los Municipios.

Por otra parte, los problemas de carácter local, sobre todo en las áreas metropolitanas derivan, en muchos aspectos, de factores que deben ser considerados en escala regional o aún nacional.

Los problemas de desarrollo urbano y su gran implicación el problema de la vivienda, deben ser estudiados con esa visión general para lograr el equilibrio de las diversas regiones del pais y evitar o contener el desarrollo excesivo de algunas áreas o regiones en detrimento de otras.

Esas tendencias y necesidades que podrian llevar a una centralización extrema, peligrosa para la subsistencia de valores humanos y que quebraría los supuestos del régimen federal de gobierno, deben ser considerados por la Nación, las Provincias y los Municipios, para encauzarlas y reducirlas a sus limites precisos, aceptando que cada una de dichas Entidades, en un orden lógico de jerarquías, cuando sea necesario y de acuerdo con las otras, cumpla la función más adecuada a su naturaleza y a su ubicación en ese orden, función que debe tener principalmente un sentido de descentralización técnica cuando se trate de planes generales. Así se podrán atenuar los factores determinantes de la crisis del federalismo argentino (20).

(20) Con acierto Zorraguf́n Becú propone la transformación del federalismo argentino, dando a la autonomia provincial un sentido funcional y económico. Es decir, que en tanto la Nación tendria las funciones politicas en su más alto sentido, de dirección del país, las Provincias se encargarian del cumplimiento o realización de los planes nacionales, con un realista fortálecimiento de sa vida propia (op. cit., págs. 246 y sigts.). 
Los Gobiernos nacional y provinciales no deben prescindir en sus planes y proyectos de la consideración y ubicación del Municipio en aquéllos. Así toda la politica de uso del suelo y de la solución del problema de la vivienda, afecta e interesa a los Municipios. Debe preocuparle a los gobiernos tener una visión de conjunto desde arriba, de los problemas de vida municipal, en cuanto se inserten en los generales del Estado. Les interesa que los Municipios lleven una vida sana y eficaz para que sean elementos activos, de verdadera cooperación en la solución de los problemas de todo el país, y que coordinen sus actividades con las del Estado.

Este criterio con vigencia en toda organización política, es especialmente más necesario en países federales, puesto que la idea del régimen y el gobierno municipal se inserta necesariamente en la idea de descentralización que supone la forma federal de gobierno. Una anemia del Municipio strpone debilitamiento de la energía de la vida provincial. Para que las. Provincias sean Entidades robustas en el organismo equilibrado de la Nación han de estar compuestas por Municipios. con plenitud de vida y de acción, y a las que no se prive de funciones que les son propias de acuerdo a su naturaleza.

Es decir, que dentro de la forma federal de gobierno, se puede conciliar perfectamente la necesidad de respetar las autonomías provinciales y municipales con las conveniencias de una planificación de alcance nacional en el desarrollo de muchos aspectos de la vida de la comunidad.

De acuerdo con tal criterio, podría existir, sin objeción cie carácter constitucional, organismos nacionales de asesoramiento y cordinación en la solución de los problemas de planificación y de vivienda. El Gobierno federal también podría prestar asistencia técnica y financiera a los Municipios para los estudios sobre la materia en el orden local.

Por su parte, las Provincias y los Municipios podrian cum- 
plir mediante acuerdos entre sí y con el Gobierno federal, una función de descentralización técnica en el cumplimiento de las planificaciones de carácter general, regional o metropolitano; en forma tal que no se vulneren las autonomias y que, por el contrario, dichas planificaciones cuenten con la conformidad deliberada y activa de las Entidades locales. 